\title{
Chemical kinetics studies at high temperatures using shock tubes
}

\author{
B. Rajakumar, ${ }^{a}$ D. Anandraj, ${ }^{a}$ K. P. J. Reddyb and E. Arunan ${ }^{a *}$
}

"Department of Inorganic and Physical Chemistry, ${ }^{b}$ Department of Aerospace Engineering, Indian Institute of Science, Bangalore 560 012, India.

email: arunan@ipc.iisc.ernet.in

Abstract

Shock tube is an unique facility to create temperature gradients exceeding million degrees Kelvin per second. We have established two shock tubes for measuring the kinetic reaction rates at high temperatures with two different but complementary detection techniques. The first one is a single pulse shock tube, in which the reflected shock is used to heat the molecules. The equilibrated products are analyzed by gas chromatograph and infrared spectrometer. The second one uses laser-schlieren system for online monitoring of the chemical reactions behind primary shock wave. The details of the shock tubes and some typical results obtained are presented in this paper.

Keywords: Chemical kinetics, shock tube, shock wave, laser-schleiren technique.

\section{Introduction}

Chemical kinetics has come a long way since Arrhenius found out the empirical relation between the rate constant and temperature [1]. Today, physical chemists do research in state-to-state kinetics in which the rates are measured for reactions with a reactant prepared in a single quantum state transforming into a product in a single quantum state. State-to-state kinetics is very valuable for determining the potential energy hyper-surface that controls the reaction and for fundamental understanding in reaction dynamics [2]. However, the need for temperature-dependent rate constant $k(T)$ in practical systems such as combustion, explosion, detonation, etc. is pertinent even today. Chemists have always been looking for ways to improve the performance of a fuel, a propellant or a fire-retardant. Ethanol has been used as a fuel additive for over 20 years in many countries [3] and recently the Government of India has also issued a directive to add it to petroleum products to be used as fuel in automobiles. Also, the effective fire-retardant methyl bromide (halon) and aerosol propellants (freons) have to be replaced as they cause stratospheric ozone depletion [4]. Naturally, as we learn more about the nature of the chemicals used in these commercial applications, there will be a need to develop alternatives. It will be important to study these chemicals in the laboratory under conditions pertinent to the real situation. In this regard, shock tubes offer unique features to enable such studies in the laboratory.

Shock tube is by no means a modern technique. It has been used for the last several decades with applications in chemistry, physics, material sciences, and aerodynamics and biology, as well. International biennial symposia are held on the development and applications of shock tube technology and the proceedings [5] from the symposia are useful sources of references. A comprehensive three-volume handbook has been produced in 2000 [6]. There is a vast body of 
literature available not only on the design and performance of the shock tubes but also on the nature of the shock wave and its interaction with the test gas. In India, shock tube facilities have been established at a few laboratories at the Indian Institutes of Technology and the Indian Institute of Science [7]-[9]. However, so far 'chemists' have not exploited the unique capabilities of the shock tube in India. Realizing the importance of a fruitful collaboration between the Aerospace Engineering and Chemistry departments of the IISc, we have established a high-temperature chemical kinetics laboratory with two dedicated shock tubes. This paper describes the details of the newly developed facilities, highlighting the important differences between our design and the existing ones along with some typical data obtained using these facilities.

\section{Chemical (single pulse) shock tube: CST-1}

The chemical shock tube, CST-1, is an aluminium tube of $50.8 \mathrm{~mm}$ diameter. It has $1.3 \mathrm{~m}$ long driver section and $2.6 \mathrm{~m}$ long driven section separated by an aluminium diaphragm. The length of the driver and/or driven section could be varied by adding small segments (Fig.1). Two homemade platinum thin film thermal sensors, mounted $0.3 \mathrm{~m}$ apart towards the end portion of the driven section are used to measure the shock velocity. The outputs from the two sensors trigger a counter (HP 5314A) to start and stop counting. The output from one of the sensors is also used as the trigger source for the digital oscilloscope (Tektronix TDS 210) which collects the pressure signal from a piezoelectric transducer (Kistler 601A) mounted at the end of the driven section. Thus the shock velocity could be independently measured using the scope and cross-verified with that measured from the timer. Helium is used as the driver gas.

The sample is loaded into the driven section as a dilute mixture in argon at a pressure, $P_{1}$ and temperature $T_{1}$. The diaphragm is ruptured by increasing the pressure $P_{4}$ in the driver side, which creates a normal shock wave travelling through the sample gas in the driven side. At the same time, an expansion fan travels into the driver section in the opposite direction. The temperature and pressure of the sample gas is raised to $T_{2}$ and $P_{2}$ by the passage of the primary shock wave. The heated gas travels behind the normal shock wave at a slower velocity than the shock wave velocity. The contact surface separates the shocked test gas from the driver gas. The spacing

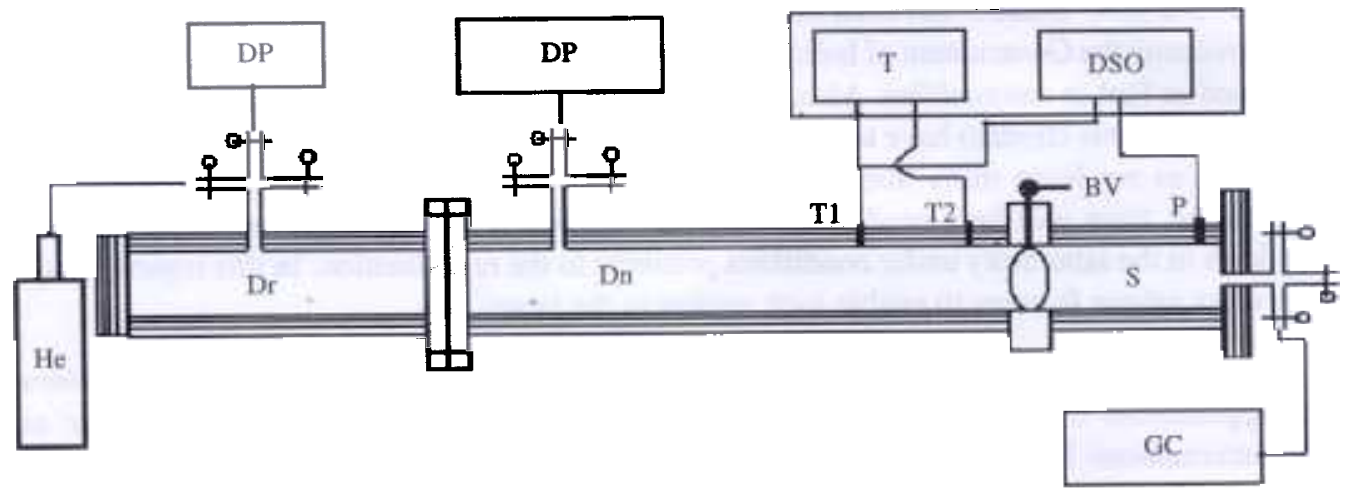

Fig. 1. Schematic diagram of the CST-1: DP-6" Diffusion pump; Dr-Driver section; DSO-Digital.storage oscilloscope Tektronix, TDS 210; Dn-driven section; S-sample chamber; T1 and T2-homemade thermal sensors; T-HP 5314A counter; P-pressure transducer, Kistler model 601A; BV-ball valve; GC-HP $6890^{\text {plus }}$ gas chromatograph with FID detector. 
between the contact surface and the shock front (i.e. the test gas region) increases along the length of the driven section. The primary shock is reflected at the end flange and the reflected shock wave further raises the temperature and pressure of the test gas to $T_{5}$ and $P_{5}$, respectively. The sample is kept at this temperature until the expansion fan passes through the contact surface, resulting in sudden drop in temperature and pressure. Cooling rate of $1 \times 10^{6} \mathrm{~K} \mathrm{~s}^{-1}$ is achieved easily. Thus, the reflected shock produces a high temperature pulse with a rise time of about $1 \mu \mathrm{s}$ and pulse length which can be varied from 0.5 to $1.5 \mathrm{~ms}$. Hence, such shock tubes are known as single pulse shock tubes (SPST). If the reflected shock wave meets the contact surface before the arrival of the expansion fan, it can lead to complicated interactions between the two depending on the impedance of the two regions. In the single pulse shock tube, the driver gas is typically much lighter (hydrogen or helium) than the driven gas (argon or krypton). It ensures that the interaction between the reflected shock and the contact surface results in an expansion wave cooling the test gas. Moreover, two attachments to the shock tube are used and they are discussed

Firstly, a dump tank is used for producing an expansion fan as well as for 'swallowing' the cold driver gas preventing the mixing of the driver and driven gases. It also prevents multiple reflection of the shock. In the original version of SPST, the dump tank was placed at the end of the driver section [10] but later on it was placed near the diaphragm in the driven section. The operation of the dump tank near the diaphragm was never fully understood [6] but the pressure trace measured near the end flange showed that the heating pulse was well defined and the cooling rate was good in the presence of the dump tank [11]. Another attachment that is common to the SPSTs is a ball valve near the end section [12]. The ball valve is essential to have a welldefined pulse length or dwell time, as it is commonly referred to in SPST literature [13].

Several calibration experiments were carried out in our laboratory to refine the design of the SPST. It was noted that with $\boldsymbol{P}_{1}$ above 350 torr and a driver to driven length ratio of about 2 , a well-defined pulse of about $600 \mu$ s could be produced near the end flange as measured by the pressure transducer. A numerical simulation using ideal one-dimensional shock relations [14] was also carried out for our shock tube conditions. It was found that the sample gas in the $2.6 \mathrm{~m}$ long driven section was compressed by the primary shock wave to about $0.8 \mathrm{~m}$ long region, i.e. the distance between the contact surface and the shock front was $0.8 \mathrm{~m}$ when the primary shock wave reaches the end flange. This is the primary reaction zone. The computed pressure jumps due to the primary and reflected shock waves at locations $3.5,3.7,3.85$ and $3.9 \mathrm{~m}$ in the shock tube are shown in Fig. 2 (The distance measured from left to right in Fig. 2. The diaphragm is at $1.3 \mathrm{~m}$ and the end flange is at $3.9 \mathrm{~m}$ ). As is evident from this figure, the pulse length varies from a maximum at the end flange to nearly zero within the primary reaction zone, depending on the location at which the expansion fan meets the reflected shock. Even at $3.5 \mathrm{~m}$, well within the primary reaction zone, the reflected shock arrives after the expansion fan and the sample in this region never experiences the reflected shock. It is clear that a significant fraction of the sample gas in the primary reaction zone will not feel the reflected shock at all. In our design, the ball valve is placed $0.3 \mathrm{~m}$ from the end flange and the sample gas is loaded only in this section. The region between the ball valve and the diaphragm is filled with argon only to a slightly larger pressure. The ball valve is opened seconds before rupturing of the diaphragm. The sample gas in this section is compressed to within $9 \mathrm{~cm}$ from the end flange and the pressure transducer is 


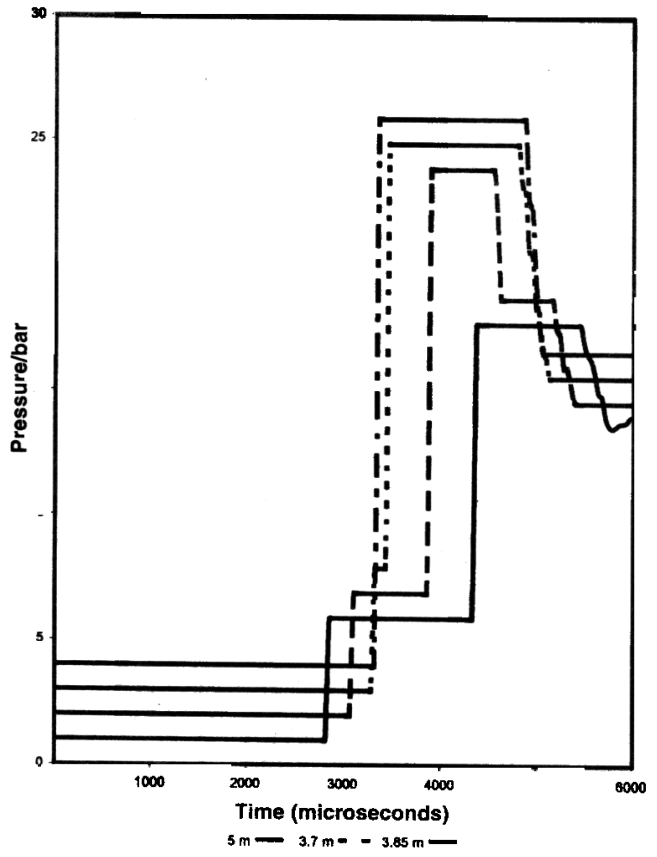

Fig. 2. Simulated pressure changes occurring at various locations within the primary reaction zone in the shock tube as the primary and reflected waves travel through the tube. The driver section is $1.3 \mathrm{~m}$ and the driven section is $2.6 \mathrm{~m}$. The legend gives the location of the pressure measurement. The end flange is at $3.9 \mathrm{~m}$ where the dwell time is maximum. Experimental measurement (in Fig. 3) corresponds to $3.85 \mathrm{~m}$. For clarity, the pressure traces at $3.7,3.85$ and $3.9 \mathrm{~m}$ have been displaced by 1,2 and 3 unit(s) in the $y$-axis, respectively.

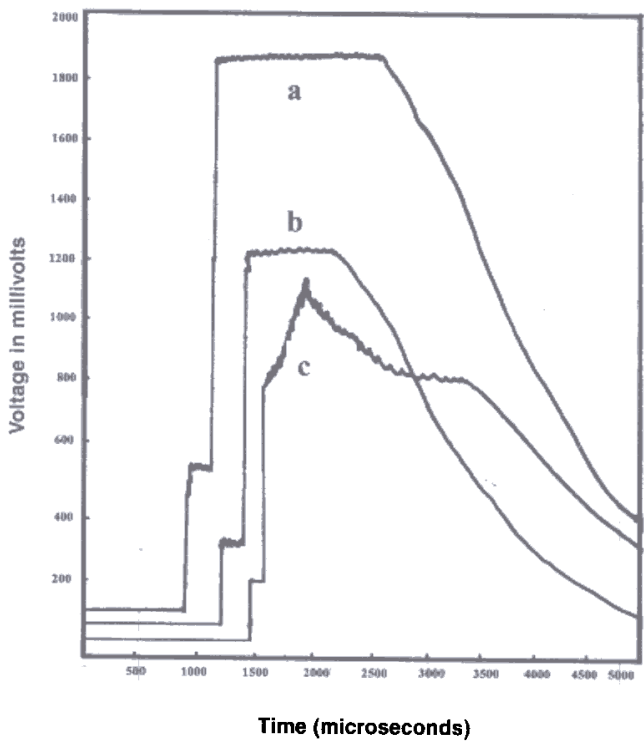

Fig. 3. Experimental pressure traces with (a) longer ( $2 \mathrm{~m}$ ) driver section and $P_{1}=450$ torr with a dwell time of 1.38 $\mathrm{ms}$, (b) shorter driver section $(1.3 \mathrm{~m})$ and $P_{1}=450$ torr with a dwell time of $0.72 \mathrm{~ms}$, and (c) $P_{1}=100$ torr with shorter driver section. In ' $c$ ', multiple reflections occur before the quenching by expansion fan and hence the dwell time and $P_{5}$ are not well defined.

located at the center of this region. Hence, the dwell time measured by the transducer is very close to the reaction time. Three pressure traces from different experiments are shown in Fig. 3. At higher $P_{1}$ (450 torr), well-defined heat pulses of (a) 1.37 and (b) $0.72 \mathrm{~ms}$ are clearly evident in the figure. The length of the driven section for trace ' $a$ ' was $2 \mathrm{~m}$ and that for trace ' $b$ ' $1.3 \mathrm{~m}$. Comparing Figs 2 and 3, it is clear that the experimental results closely follow the simulated results qualitatively. A pressure trace taken at $P_{1}=100$ torr ( ' $c$ ' in Fig. 3) shows that neither dwell time nor temperature is well defined. Here, the reflected shock travels through the reaction zone and meets the contact surface before the arrival of the expansion fan. Moreover, the primary shock does not seem to have been fully formed. Under these conditions, the sample is not heated uniformally leading to undefinable temperature and dwell time.

In SPST experiments, an accurate estimate of $T_{5}$ is essential for measuring reliable kinetic data. However, the ideal shock relations [15] used for determining reflected shock properties are not valid due to several reasons such as real gas effects, boundary layer problems, exo/ endothermicity of the chemical reactions, etc. Chemists have established a convenient way to work around this problem. It is known as the internal standard method. In this method, a molecule 
Table I

The temperature behind reflected shock wave $\left(T_{5}\right)$ determined using three different methods

\begin{tabular}{llll}
\hline No. & $T_{5}\left(M_{\mathrm{s}}\right)^{\mathrm{a}}$ & $T_{5}\left(M_{\mathrm{r}}\right)^{\mathrm{b}}$ & $T_{5}$ (Kinetic $^{\mathrm{c}}$ \\
\hline 1 & 1068 & 1000 & 991 \\
2 & 1094 & 1035 & 1024 \\
3 & 1146 & 1056 & 1031 \\
4 & 1153 & 1087 & 1060 \\
5 & 1157 & 1082 & 1061 \\
6 & 1236 & 1138 & 1104 \\
7 & 1236 & 1138 & 1107 \\
8 & 1261 & 1172 & 1125 \\
9 & 1325 & 1232 & 1171 \\
10 & 1384 & 1278 & 1210 \\
\hline
\end{tabular}

a. Calculated from measured incident shock velocity and ideal shock relations. b. Calculated from measured incident and reflected shock velocity without any assumptions about the particle velocity in the reflected zone (Ref.12). c. Determined using the kinetic parameters for $\mathrm{CH}_{3} \mathrm{CH}_{2} \mathrm{Cl} \rightarrow \mathrm{C}_{2} \mathrm{H}_{4}+\mathrm{HCl}$ reaction from ref. 18 .

for which the kinetic parameters are well known is subjected to shock waves along with the molecule of interest. By comparing the extent of reaction for the two molecules, the relative rates can be determined accurately. Recently, the possibility of using an external standard has been tested in our laboratory [16] and at the University of Illinois at Chicago [17]. Table I shows $T_{5}$ values calculated using the measured primary shock velocity, primary and reflected shock velocities and the external standard method. Both $T_{2}$ and $T_{5}$ can be calculated from the measured primary shock velocity, $u_{1}$, using the Rankine-Hugoniot equations $\left(T_{i j}\right.$ implies $\left.T_{i} / T_{j}\right)$ [15]:

$$
\begin{gathered}
T_{21}=\frac{\left(\gamma M_{1}^{2}-\frac{\gamma-1}{2}\right)\left(\frac{\gamma-1}{2} M_{1}^{2}+1\right)}{\left(\frac{\gamma+1}{2}\right)^{2} M_{1}^{2}} ; \\
T_{51}=\frac{\left\{2(\gamma-1) M_{1}^{2}+(3-\gamma)\right\}\left\{(3 \gamma-1) M_{1}^{2}-2(\gamma-1)\right\}}{(\gamma+1)^{2} M_{1}^{2}} .
\end{gathered}
$$

Here, $\gamma$ is the specific heat ratio for the test gas, and $M_{1}$ the shock Mach number defined as the ratio of the primary shock velocity and the speed of sound in test gas, $a_{1}$ i.e. $M_{1}=u_{1} / a_{1}$. As pointed out earlier and evident from Table I, this method of calculating $T_{5}$ leads to large errors. Tschuikow-Roux has shown that $T_{5}$ can be estimated from the following equations using measured primary shock velocity $u_{1}$ and measured reflected shock velocity $u_{2}$ without any assumptions:

$$
\begin{aligned}
& M_{1}=\left[\beta_{1}\left(1+\alpha_{1} P_{21}\right)\right]^{1 / 2} ; \\
& T_{21}=\left[\frac{P_{21}\left(\alpha_{1}+P_{21}\right)}{1+\alpha_{1} P_{21}}\right]^{1 / 2} ; \\
& M_{2}=\left[\beta_{1}\left(1+\alpha_{1} P_{52}\right]^{1 / 2} ;\right.
\end{aligned}
$$




$$
T_{52}=\frac{P_{52}\left(\alpha_{1}+P_{52}\right)}{\left(1+\alpha_{1}+P_{52}\right)}
$$

Here, $\alpha_{1}=\left(\gamma_{1}+1\right) /\left(\gamma_{1}-1\right)$ and $\beta_{1}=\left(\gamma_{1}-1\right) / 2 \gamma_{1}$. As is evident from Table I, this method of evaluating $T_{5}$ is better than the one using the primary shock velocity only. It is closer to the kinetic temperature measured using a standard chemical reaction. Table I includes results from such measurements which used 1,2-HCl elimination from ethyl chloride (EC) as the standard reaction. In this method, $T_{5}$ is defined as:

$$
T_{5}=\frac{E_{a}}{R \ln \left(A / k_{1}\right)} .
$$

Arrhenius parameters $\left(A\right.$ and $\left.E_{a}\right)$ for this reaction are well established [18] and measuring the EC by gas chromatograph before and after the experiment gives $k_{1}$.

The SPST set-up can be used to measure the thermal rate parameters of chemical reactions between 800 and $1500 \mathrm{~K}$ for dwell time ranging from 0.5 to $1.5 \mathrm{~ms}$. The CST-1 has been used recently to study the thermal decomposition of 1,2-dichloroethane which is an important industrial process for producing vinyl chloride [16]. It is for the first time that the kinetics of this reaction was studied without complication arising from wall collisions. Currently, thermal decomposition of haloethanols such as $\mathrm{FCH}_{2} \mathrm{CH}_{2} \mathrm{OH}$ and $\mathrm{ClCH}_{2} \mathrm{CH}_{2} \mathrm{OH}$ is being studied. These can decompose by $\mathrm{HF} / \mathrm{HCl}$ elimination, $\mathrm{H}_{2} \mathrm{O}$ elimination or $\mathrm{C}-\mathrm{C}$ dissociation.

\section{Chemical shock tube-CST-2 and laser-schlieren facility}

According to Kiefer "of all the various diagnostics devised for kinetic studies in shock waves, laser-schlieren technique makes perhaps the greatest use of the special nature and capabilities of the shock tube" [19]. In the SPST, the kinetics is deduced indirectly by following the equilibrated products after the heat pulse produced by the reflected shock wave. As all the products can be analyzed, it gives a comprehensive outlook for the reaction. However, none of the reactant, product or intermediate is followed in real time. In contrast, the laser-schlieren technique is a real-time technique. It is based on the fact that a ray of light traversing any medium having a refractive index gradient normal to the propagation direction will be deflected. Endothermic reactions initiated by shock heating lead to an axial density gradient in the shocked gas. Under typical conditions of the experiment, this density gradient is directly proportional to the angle of deflection. It is also linearly related to the rate of reaction in the post-shock flow [19]. Thus, measuring the deflection of a light ray as the primary shock passes through the observation window leads to the rate of the physical/chemical change directly. Almost all the laboratories that have developed the laser-schlieren technique use the output of a He-Ne laser for measuring the deflection. In this technique, the primary shock wave is used for instantly heating the molecules to temperatures in a wide range from 360 to $3600 \mathrm{~K}$. The time scale is mueh smaller, typically 10 $\mu \mathrm{s}$ or less.

The CST-2 in our laboratory is made of stainless steel tube of diameter $39 \mathrm{~mm}$. It has $1 \mathrm{~m}$ long driver and $3 \mathrm{~m}$ long driven sections. Neither a ball valve nor a dump tank is needed as only 
the primary shock is used for heating and the observation is made in real time. The physical/ chemical change is followed in the primary reaction zone at $P_{2}$ and $T_{2}$ for $10 \mu \mathrm{s}$ only and hence the reflected wave and all the other complications do not affect the experiment. An optical window is placed at the center of a separate section of the shock tube that is $0.2 \mathrm{~m}$ long. Two pressure transducers $(0.2 \mathrm{~m}$ apart) and two thermal sensors $(0.5 \mathrm{~m}$ apart) are used for measuring the shock velocity. The pressure transducers are mounted on either side of the observation window to record the pressure history as well. The thermal sensors are mounted in the last section of the shock tube. It is important to ascertain that the shock wave is fully formed but has not started deteriorating at the observation point. The schematic diagram of the CST-2 is shown in Fig. 4.

The output from the He-Ne laser at $632.8 \mathrm{~nm}$ (maximum power $10 \mathrm{~mW}$ ) passes through the shock tube and falls on a quadrant photodiode in which the quadrants are paired. The diameter of the beam is $1 \mathrm{~mm}$ at the windows. The signal from the quadrant diode is differentially amplified and fed to an oscilloscope (Tektronix, TDS 230). The detailed electronic circuit used for the detection set-up is given in Fig. 5. The path length is about $4 \mathrm{~m}$ and the laser beam nearly fills the active area of the diode, which is $2 \mathrm{~mm}$. Before the shock wave arrives, both pairs of the diodes get the same signal and the differential amplifier gives a null signal. As the shock wave travels through the observation window, the laser beam is deflected following any density/refractive index gradient. From this signal the rate of the physical/chemical change can be directly determined. Rotatable mirror is used for calibrating the voltage signal as it can provide wellcharacterized angular deflections.

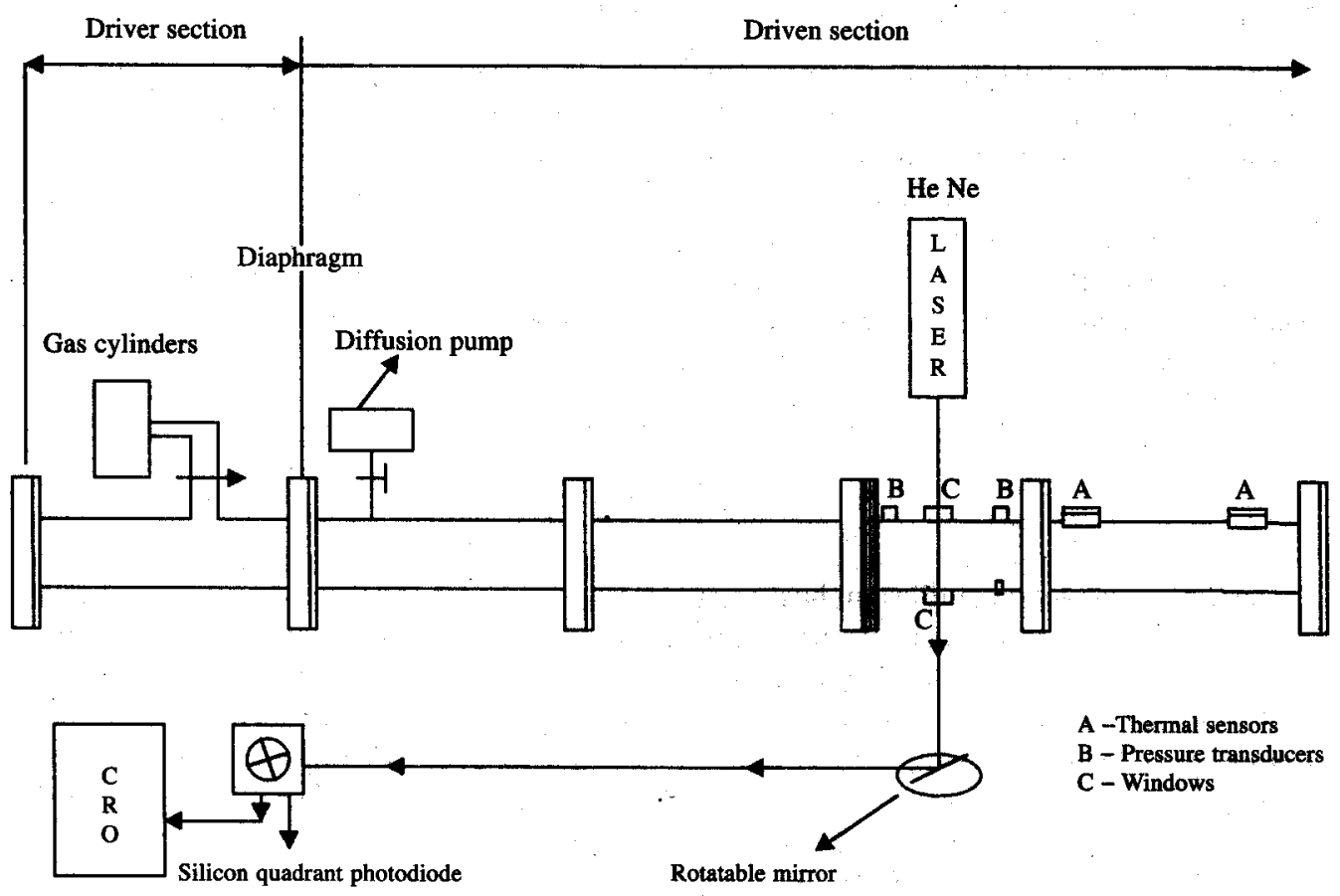

Fig. 4. Schematic diagram of the CST-2 with laser-schlieren detection. 


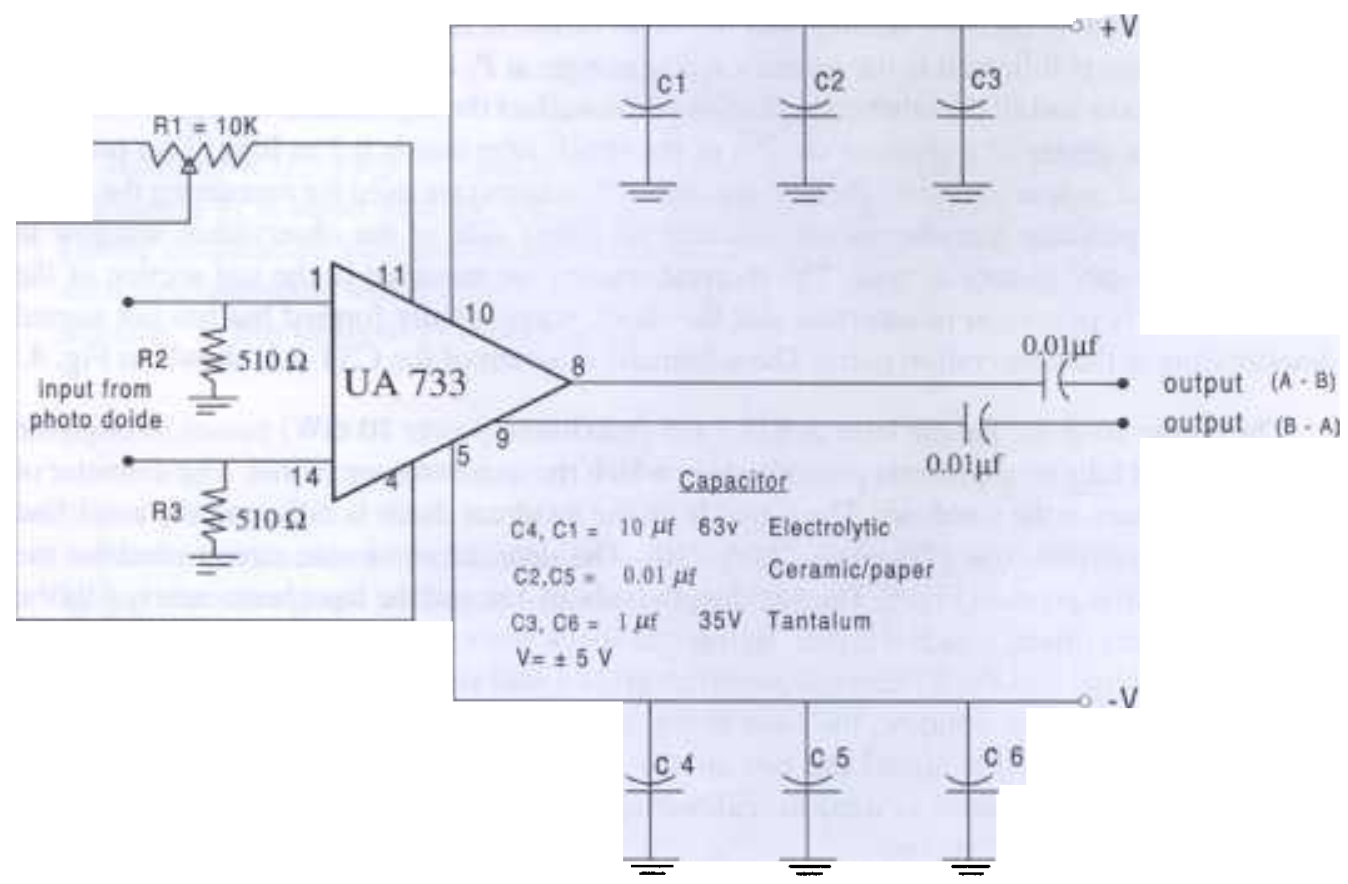

Fig. 5. Electronic circuit diagram for the detection set-up in the laser-schlieren experiment.

Several experiments have been carried out to test the operation of the CST-2 and the laserschlieren system. The schlieren signal observed with pure argon (not shown) in the driven section was in excellent agreement with earlier report [20]. This method has been used for measuring rates of vibrational relaxation and that of chemical reactions [19]-[22]. The schlieren signal observed in $\mathrm{O}_{2}$ is shown in Fig. 6. Kiefer has discussed in detail the procedure for extracting vibrational relaxation time, $\tau$, from this signal which gives the laboratory relaxation time, $\tau_{1}[19]$. Usually, the quantity $P \tau$ is reported where $p$ is the pressure. It depends only on temperature and composition.

$$
P \tau=P \tau_{l} \frac{C_{P}}{C_{p}^{*}}\left(\frac{\rho}{\rho_{o}}\right)
$$

Here, $C_{P}$ is the total equilibrium heat capacity, $C_{P}^{*}$ is that with vibration frozen, $\rho$ and $\rho_{\mathrm{o}}$ are the equilibrium and initial density. The schlieren oscillogram shown in Fig. 6 was obtained at $P=0.62 \mathrm{~atm}$ and $T=2008 \mathrm{~K}$. The semi-log plot from this signal is shown in Fig. 7. It is fairly linear (correlation coefficient 0.99 ) but there is evidence for some disturbances in the shock front at later times leading to poorer S/N. A bi-exponential fit does not improve the correlation coefficient. The $P \tau$ is determined to be $8.2 \times 10^{-6} \mathrm{~atm} \mathrm{~s}$ in reasonable agreement with earlier measurements $\left(6.4 \times 10^{-6} \mathrm{~atm} \mathrm{~s}\right)$ [19]. The schlieren signals obtained with pure $\mathrm{Ar}$ and $\mathrm{O}_{2}$ confirm that the experimental set-up is fully operational.

The laser-schlieren system is currently being used to study C-I bond-breaking reactions of 


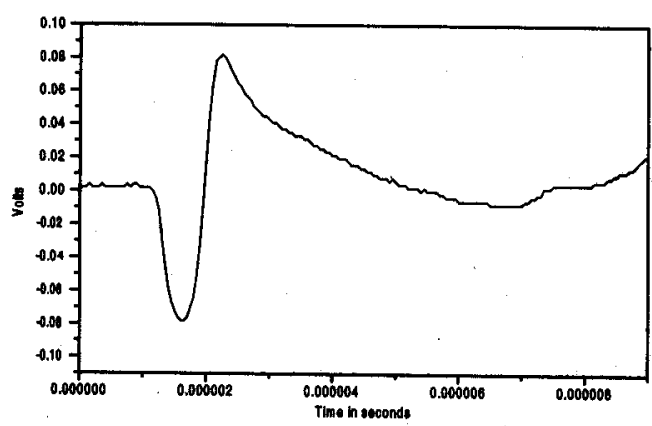

Fig. 6. Laser-schlieren signal from $\mathrm{O}_{2}$ at $T=2006 \mathrm{~K}$ and $P=0.65 \mathrm{~atm}$. The initial oscillation is due to the arrival of the shock wave after which the signal shows a single exponential decay corresponding to vibrational relaxation. Laboratory time zero is at the peak of the signal.

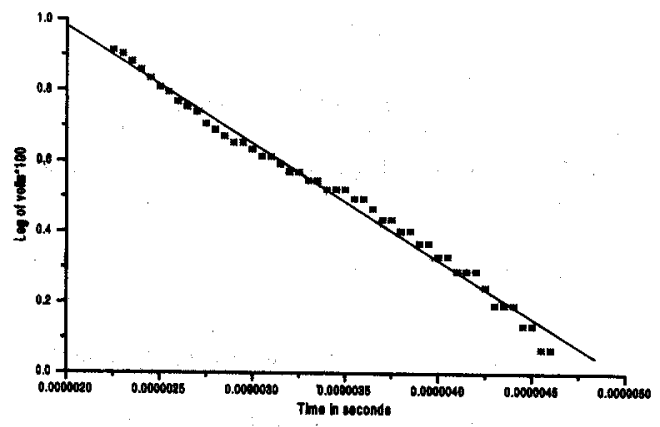

Fig. 7. Semi-log plot of the voltage versus laboratory time for the oscilloscope trace shown in Fig. 6.

iodoalokanes such as $\mathrm{CH}_{2} \mathrm{ClI}$, which are important in the atmosphere. The chloroiodomethane, $\mathrm{CH}_{2} \mathrm{ClI}$, happens to be the second largest source of $\mathrm{I}$ atoms in the troposphere next only to $\mathrm{CH}_{3} \mathrm{I}$ [23]. There have been numerous studies on this interesting molecule in the past few years [24]. The CST-2 will be used for studying the kinetics of reactions that are of interest in combustion and atmospheric chemistry.

In laser-schlieren experiments, only the density gradient is measured and no information about the exact chemicals/reactions is obtained. Hence, the reaction mechanism should be independently established. If more than one reaction is contributing to the density gradient, computer simulation will be required to determine the kinetic parameters of all the reactions. In closing, reference is made to a comprehensive review on kinetic studies in shock tube that appeared earlier this year [25].

\section{Conclusions}

Active collaboration between the Departments of Aerospace Engineering and Inorganic and Physical Chemistry has led to the development of a high-temperature chemical kinetics laboratory at the Indian Institute of Science. Two shock tubes have been established that are complementary in nature. One is designed as a single pulse shock tube, which is useful for comprehensive chemical kinetics studies with a reaction time in milliseconds. The other uses real-time laser-schlieren detection technique and can be used for studying faster physical/chemical changes in microsecond time scale. Both are useful for simulating real-life conditions as in combustion, explosion or detonation, wherein only a temperature can be defined.

\section{Acknowledgement}

The authors thank the Director, IISc, and the IISc-ISRO Space Technology Cell for partial support in establishing the high-temperature chemical kinetics laboratory. They also acknowledge fruitful discussions with Profs G. O. Thomas, A. Lifshitz, W. Tsang, and J. Kurian. Messrs S. Maiti and Kiran assisted in the electronics set-up for the laser-schlieren detection system. 


\section{References}

K . J. Laidler, Chemical kinetics, Harper and Row (1987).

R. D. Levine and R. Bernstein, Molecular reaction dynamics and chemical reactivity, Oxford University Press, (1987).

A. E. Wheals, L. C. Basso, D. M. G. Alves and H. V. Amorim, Fuel ethanol after 25 years, Trends Biotechnol. 17, 482-487 (1999).

A. McCulloch, CFC and halon replacements in the environment, J. Fluorine Chem., 100, 163-173 (1999).

G. J. Ball, R. Hillier and G. T. Roberts (eds), Proc. $22^{\text {nd }}$ Int. Symp. on Shock Waves, University of Southampton Press (1999).

6. G. Ben-Dor, O. Igra, T. Elperin and A. Lifshitz (eds), Handbook of shock waves (3 volumes), Academic Press, (2000).

J. Kurian and A. K. Sreekanth, Laser-schlieren study of vibrational relaxation of molecular nitrogen by water, Chem. Phys. Lett., 114, 295-303 (1987).

8. K. Thyagarajan and K. A. Bhaskaran, Effect of argon dilution on the pre-ignition oxidation kinetics of benzene, Int. J. Energy Res., 15, 235-248 (1991).

9. G. Jagadeesh, N. M. Reddy, K. Nagashetty and K. P. J. Reddy, Forebody convective hypersonic heat transfer measurements over large angle blunt cones, J. Spacecraft Rockets, 37, 137-139 (2000).

10. H. S. Glick, J. J. Klein and W. Squire, Single pulse shock tube studies of the kinetics of the reaction $\mathrm{N}_{2}+\mathrm{O}_{2} \Leftrightarrow 2$ NO between 2000-3000 K, J. Chem. Phys., 27, 850-857 (1957).

1. A. Lifshitz, S. H. Bauer and E. L. Resler, Jr, Studies with a single pulse shock tube. I. The cis-trans isomerization of butene-2, J. Chem. Phys., 38, 2056-2063 (1963).

12. E. Tschuikow-Roux, J. M. Simmie and W. J. Quirring, A re-examination of the single pulse shock tube technique: Determination of reflected shock temperature, Astronaut. Acta, 15, 511-521 (1970).

13. E. Tschuikow-Roux, Reaction dwell time and cooling rate in a single pulse shock tube, Phys. Fluids, 8, 821-825 (1965).

14. G. O. Thomas, Fortran program rcmblast.for for simulation of shock and detonation waves. Implementation based on' Gottlieb, J. J., J. Comput. Phys., 78, 160-177 (1988).

15. A. G. Gaydon and I. R. Hurle, The shock tube in high temperature chemical physics, Reinhold (1963).

16. B. Rajakumar, K. P. J. Reddy and E. Arunan, Unimolecular $\mathrm{HCl}$ elimination from 1, 2-dichloroethane: A single pulse shock tube and ab initio study, J. Phys. Chem. A, 2002 (in press).

17. R. S. Tranter, R. Sivaramakrishnan, N. Srinivasan and K. Brezinsky, Calibration of reaction temperatures in a very high pressure shock tube using chemical thermometers, Int. J. Chem. Kinetics, 33, 722-731 (2001).

18. P. J. Evans, T. Ichimura and E. Tschuikow-Roux, A comparison of two single pulse shock tube techniques: The thermal decomposition of ethyl chloride and $n$-propyl chloride, Int. J. Chem. Kinetics, 10, 855-869 (1978).

19. J. H. Kiefer, Laser-schlieren technique in shock tube kinetics. In Shock waves in chemistry (A. Lifshitz, ed.), Marcel Dekker (1981).

20. J. H. Kiefer and R. W. Lutz, Vibrational relaxation of deuterium by a quantitative schlieren method, J. Chem. Phys., 44, 658-666 (1966). 
21. J. H. Kiefer and S. S. Kumaran, Rate of $\mathrm{CH}_{4}$ dissociation over $2800-4300 \mathrm{~K}$ : The low pressure limit rate constant J. Phys. Chem., 97, 414-420 (1993).

22. J. H. Kiefer, Densitometric measurements of the rate of carbon dioxide dissociation in shock waves, J. Chem. Phys., 61, 244-248 (1974).

23. R. Vogt, R. Sander, R. von Glasow and P. J. Crutzen, Iodine chemistry and its role in halogen activation and ozone loss in the marine boundary layer: a model study, J. Atmos. Chem., 32, 375-395 (1999).

24. E. Arunan, S. P. Vijayalakshmi, R. Valera and D. W. Setser, Infrared chemiluminescence: Evidence for adduct formation in the $\mathrm{H}+\mathrm{CH}_{2} \mathrm{XI}$ reaction and studies on $\mathrm{N}+\mathrm{CH}_{2} \mathrm{X}(\mathrm{X}=\mathrm{Cl} / \mathrm{F} / \mathrm{I} / \mathrm{H})$ reactions, Phys. Chem. Chem. Phys., 4, 51-60 (2002).

25. K. A. Bhaskaran and P. Roth, The shock tube as wave reactor for kinetic studies and material systems, Progr. Energy Combustion Sci., 28. 151-192 (2002). 